\title{
1 Overcoming barriers to the registration of new varieties
}

2 Chin Jian Yang ${ }^{1}$

3 Joanne Russell

$4 \quad$ Luke Ramsay²

5 William Thomas ${ }^{2}$

$6 \quad$ Wayne Powell ${ }^{1}$

$7 \quad$ lan Mackay ${ }^{1,3}$

8 'Scotland's Rural College (SRUC), Kings Buildings, West Mains Road, Edinburgh, EH9 3JG, 9 UK.

10 'The James Hutton Institute, Invergowrie, Dundee, DD2 5DA, UK.

11 3IMplant Consultancy Ltd., Chelmsford, UK.

12

13 Corresponding author:

14 lan Mackay, i.j.mackay@gmail.com 


\section{Abstract}

16 Distinctness, Uniformity and Stability (DUS) is an intellectual property system introduced in 1961

17 by the International Union for the Protection of New Varieties of Plants (UPOV) for safeguarding

18 the investment and rewarding innovation in developing new plant varieties. Despite the rapid 19 advancement in our understanding of crop biology over the past 60 years, the DUS system has

20 not changed and is still dependent upon a set of morphological traits for testing candidate 21 varieties. As the demand for more plant varieties increases, the barriers to registration of new 22 varieties become more acute and thus require urgent review to the system. To highlight the 23 challenges and remedies in the current system, we evaluated a comprehensive panel of 805 UK 24 barley varieties that span the entire history of DUS testing. Our findings reveal the system 25 deficiencies and provide evidence for a shift towards a robust genomics enabled registration 26 system for new crop varieties. 


\section{Introduction}

Crop breeding involves considerable investment of time, resources and money by seed companies to produce improved varieties of plants. Plant Variety Rights (PVR) is a form of intellectual property introduced in 1961 by the International Union for the Protection of New Varieties of Plants (UPOV) to protect the breeders' investment in creating new varieties, support innovation and serve market demand ${ }^{1}$. For almost 60 years, the protection of new plant varieties through the award of PVR relied on passing two tests: Distinctness, Uniformity and Stability (DUS), and Value for Cultivation and Use (VCU). DUS requires the new variety to be distinct from the common knowledge varieties, uniform across seeds that constitute the variety, and stable across environments ${ }^{2}$. DUS is usually defined by a set of morphological traits, although isozyme electrophoresis and molecular markers are occasionally used ${ }^{3}$. On the other hand, VCU requires the new crop variety to demonstrate improvement in yield, biotic or abiotic resistance, and quality characteristics $^{4}$. Unlike VCU traits such as yield and disease resistance that have been the centre of attention in crop breeding ${ }^{5}$, the DUS system has received relatively little attention despite its pivotal role in the registration of new varieties ${ }^{6,7}$.

The pressure on the current DUS system stems from multiple issues. As more new varieties arise, the DUS trait combinatorial space becomes more limited and requires additional effort in breeding unique DUS trait combinations. Many DUS traits have low heritabilities ${ }^{7}$ which means more trait variability due to environmental fluctuations and limited reliability of DUS trait scores outside of the trial environment. While the current system is well established for major crops, it is hard to implement in minor or orphan crops since the traits for DUS are hard to determine $^{8,9}$. Furthermore, the current DUS system is largely designed for inbred species or varieties which is hardly practical in outbreeding species or hybrid varieties ${ }^{10}$. Lastly, there is also a lack of definition between new varieties and essentially derived varieties (EDVs) in the current system.

Over the years, many attempts at improving the DUS system have met with little success. Suggestion for the use of molecular markers in DUS traces back to at least 1990 using minisatellites in soft fruits ${ }^{11}$. Since then, more molecular markers have been proposed for DUS, for examples, 28 SSR markers in maize ${ }^{12}, 25$ SNP markers in barley ${ }^{13}$, and 5 SSR markers in rice $^{14}$. However, the number of available DUS markers that have been proposed thus far is too few and low throughput. More recently, larger marker sets using SNP arrays have been suggested, including 3,072 SNP markers in maize ${ }^{15}$ and 6,000 SNP markers in soybean ${ }^{16}$. As of now, none of these have been officially adopted by the UPOV. Instead, UPOV sanctions the use 
of molecular markers only when they correlate with the DUS traits perfectly ${ }^{17}$, which is often possible only for a small subset of traits.

Using the UK barley DUS system as a test case (panel of 805 spring, winter and alternative barley varieties that have been accepted into the UK national list (NL), as well as 28 DUS traits), we demonstrate both the challenges and opportunities for the creation of a new DUS system.

\section{Results}

The 28 barley DUS traits include seasonal type and 27 above-ground morphologies, including leaves, ears and spikelets (Table 1). Currently within the UK, barley DUS trait data are publicly available from the National Institute of Agricultural Botany (NIAB) in England and the Science and Advice for Scottish Agriculture (SASA) in Scotland. We obtained the data from these two sources, and supplemented with additional data from ${ }^{7}$. The NIAB data serves as our primary data as it is more complete than the SASA data, which was only used for comparative analysis. 21 DUS traits are scored on a scale of 1 to 9 or smaller subset of the scale, and seven traits are scored on a binary scale (Table S1). Of the 27 traits excluding seasonal type, two traits are not segregating in spring barley and one in winter barley (Fig. S1). The missing rate in the DUS trait data ranges from 0 to $78 \%$, with only 5 traits above $10 \%$. In addition, our analysis included marker data for 805 varieties from the IMPROMALT collection (http://www.barleyhub.org/projects/impromalt/), of which 710 had DUS trait data.

Comparison across DUS trait scoring organisations (NIAB vs. SASA) showed an average consistency in two-third of the DUS trait scores (Fig. 1a \& 1b). In most cases, the trait score differences are small (Fig. 1a \& Fig. S2). These differences are expected given that the DUS traits were scored in different environments by different DUS inspectors. There is little to no bias in trait score differences between NIAB and SASA (Fig. S2) except for trait 6 (flag leaf: glaucosity of sheath) and trait 25 (grain: spiculation of inner lateral nerves of dorsal side of lemma). On average, trait 6 is about 1 score higher in NIAB compared to SASA while trait 25 is about 1 score lower in NIAB, which may reflect the environmental effects on these traits. Regardless, with the reduction in DUS trait combinatorial space as measured by shrinkage in DUS trait Manhattan distances over time (Fig. 1c \& 1d), especially in spring barley, small trait score differences can easily complicate variety identification. Manhattan distances are the sums of absolute differences between any two variety and lower distances imply reduced variation in DUS traits among the 
another due to variations in DUS traits. Besides, the inconsistencies are present in the majority (392/395) of the barley varieties compared, which suggests that the inconsistencies are common and excludes the possibility of poor data handling by either organisation. Given the roles of the DUS system in granting PVR, a two-third consistency across organisations is inadequate and risky.

Of all 28 barley DUS traits, 15 have low heritabilities $\left(h^{2}<0.50\right)$ (Table $\left.1 \& S 2\right)$ and are thus contradictory for DUS purposes. Briefly, any phenotypic trait can be described as an expression of genetic and environmental effects, and their interactions. Therefore, traits with low heritabilities have little replicability in trait values obtained from different environments (e.g. year, location). As expected, the DUS trait inconsistencies across scoring organisations are negatively correlated (-0.67) with heritabilities (Fig. 1e). Similar results were observed when the heritabilities were calculated from spring and winter barley separately. Consequently, instead of a fair evaluation of the genetic merits underlying new varieties, the current DUS system simply determines new varieties based on environmental stochasticity.

12 out of 21 barley DUS traits have non-zero genetic correlations with yield in spring barley (Fig. 2), which risk undesirable correlated responses upon selecting for either DUS traits or yield. Non-zero genetic correlations are the hidden cost in exchange for the genetic gain in yield. In traits with low phenotypic correlations, the unintended selection for DUS traits may not be immediately apparent to breeders. For instances, both DUS trait 10 (ear: attitude) and 11 (plant: length) are negatively correlated with yield, which translates to semi-dwarf barley plants with erect ears having higher yield than tall barley plants with recurved ears. Such correlations could help define ideal crop ideotypes ${ }^{18}$, however, they are not ideal for DUS purpose because high yielding plants are more likely to be semi-dwarf with erect ears. As the genetic gain in yield increases over time $^{19}$, it is inevitable that DUS trait combinatorial space gets more limited (Fig. 1c \& 1d) due to correlated selection responses. On the other hand, selection away from DUS trait combinatorial space risks losing the genetic gain in yield. While we have only considered correlations between DUS traits and yield, there are other VCU traits that may also constrict DUS trait combinatorial space.

GWAS results showed that 14 of 28 barley DUS traits are likely regulated by few major loci and some of these loci are likely fixed in either spring or winter barley populations (Table 2). Of the total 32 GWAS loci, 30 were identified in the combined dataset (Table S3, Fig. S3), 12 in spring-only dataset (Table S4, Fig. S4) and 16 in winter-only dataset (Table S5, Fig. S5). Part of the explanations for the difference is due to the individual datasets have smaller sample size and 
thus lower power. Another reason is because some traits are not segregating or rare in either spring or winter germplasm. Examples of these traits are: 3 (lowest leaves: hairiness of leaf sheaths), 12 (ear: number of rows), 23 (grain: husk), 26 (grain: hairiness of ventral furrow) and 27 (grain: disposition of lodicules). A major QTL for trait 3 is tightly linked to $\mathrm{Vrn}-\mathrm{H} 2$, a major vernalisation locus ${ }^{20}$ while trait 12, 23 and 27 are largely monomorphic in the UK barley breeding pool due to preferences for two-rowed barley with hulled grains and clasping (collar type) lodicules. In comparison with a previous work on DUS traits GWAS $^{7}$, the number of loci increased from 16 to 32 with 12 loci in common.

In accordance to the UPOV guidelines ${ }^{17}$, molecular markers can only be used in DUS if they confer direct relationship with the DUS traits. This might work well with those 14 traits with known major loci, although there is a risk of ignoring effects from minor or exotic loci. One such example would be anthocyanin-related traits in flag leaf (trait 4) and awn (trait 8), where anthocyaninless 1 (ant1) and ant2 are segregating in winter but not spring barley varieties in the UK (Table 2). Unless the DUS markers for ant1 and ant2 are in perfect linkage with the causative polymorphisms, these markers would give misleading results if used in spring barley. To complicate this issue further, we identified a locus at ant2 for an anthocyanin-related trait in grain (trait 24) in spring barley, which may suggest an additional linked locus that is segregating in spring barley responsible for grain-only anthocyanin pigmentation. On the other hand, it is improbable to create molecular markers that would tag any of the other 14 traits without major loci.

To extend beyond locus-specific markers, a small marker set for DUS has been proposed $^{21}$ although our evaluation showed limited distinguishing power. By simulating $F_{6}$ progeny from known parent pairs, we compared the marker set from these simulated progeny to their parents, actual variety (progeny of the parent pairs) and other simulated progeny. While most of these simulated progeny remained unique in older varieties, this is not true for newer varieties (Fig. 3a, 3b \& S5), especially in spring barley. For example, LG Goddess matched perfectly with $7.5 \%$ of the simulated progeny, and its parents Octavia and Shada matched perfectly with $8.0 \%$ and $7.8 \%$ of the simulated progeny respectively (Table S6). Furthermore, $88.4 \%$ of the simulated progeny have over $1 \%$ probability of matching with other simulated progeny (Table S6). A small marker set for DUS is problematic in a crop in which genomic diversity progressively gets narrower over time. Of the total 39 markers $^{21}$, only 4 to 22 markers are segregating between the parents analysed. Besides, these markers are not randomly distributed as there are some in strong linkage disequilibrium (LD) which would not informative. 
As a follow-up, we investigated the number of markers required for proper separation of varieties in DUS and determined that approximately 500 - 1,000 markers are likely the minimum (Fig. 4a). By comparing the Manhattan distances calculated from all 28 DUS traits against a series of randomly sampled markers, the correlation between these two distances begins to plateau at about $500-1,000$ markers. The correlation maxes out at about 0.60 , which is similar to the value previously observed by Jones et al. ${ }^{10}$. This is not surprising given that the correlation depends on the DUS trait heritabilities. Manhattan distances determined from DUS traits with high heritabilities $\left(h^{2}>0.50\right)$ showed stronger correlation with Manhattan distances from the marker data than DUS traits with low heritabilities $\left(\mathrm{h}^{2}<0.50\right)$ (Fig. 4a). Additionally, the distribution variances stabilise at similar range too (Table S7), which affirms that any marker set smaller than 500 markers is insufficient.

Given the various issues we have described in the DUS system so far, the remaining option is to use genomic markers. There are multiple ways to implement genomic markers in DUS, and we will provide a simple example here using Manhattan distances, which is one of many measures of dissimilarities among varieties. Under haploid marker coding of 0 and 1 , Manhattan distance between any two varieties is equivalent to $2 \times(1$ - similarity) where similarity is measured as the proportion of exact marker matches between two varieties. Similar to the current DUS system, we will need a reference panel (common knowledge varieties set) and the genomic marker data for the reference panel. As an example, we set all 805 barley varieties as our reference panel and computed the Manhattan distances among these varieties. The distances are divided by within and across seasonal types, as the values ranged from 0.04 to 0.69 within spring barley, 0.04 to 0.87 within winter barley and 0.44 to 0.97 between spring and winter barley (Fig. 4b). To demonstrate how genomic markers work in DUS, we simulated 1,000 $\mathrm{F}_{6}$ and $\mathrm{BC}_{1} \mathrm{~S}_{4}$ progeny from two pairs of parents in spring barley. The first parent pair is Propino and Quench, which has a distance of 0.20 and thus represents the "low" distance parents. The second parent pair is Riviera and Cooper, which has a distance of 0.59 and thus represents the "high" distance parents. Given an arbitrary minimum threshold of 0.05 for distinctness, $13.0 \%$ of $F_{6}$ progeny and $59.6 \%$ of $\mathrm{BC}_{1} \mathrm{~S}_{4}$ progeny from the low parents would be rejected for lack of distinctness, while none of the $\mathrm{F}_{6}$ progeny and $4.9 \%$ of the $\mathrm{BC}_{1} \mathrm{~S}_{4}$ progeny from the high parents would be rejected 187 (Fig. 4c).

Another important consequence of using genomic markers in DUS is the regulation of essentially derived varieties (EDVs). As of current standard, the definition of EDVs is unclear and 190 it often involves complicated and expensive court proceedings to determine EDVs ${ }^{22}$. Furthermore, 
the information on whether a market variety is an EDV is not generally disclosed to public, and it is possible that no EDV ever makes it into the market. With genomic markers, any varieties submitted for DUS evaluation that failed to pass the minimum distance threshold would be considered for EDVs. If these varieties demonstrate justifiable VCUs compared to the common knowledge varieties, then EDVs should be granted. Curiously, among the varieties in our reference panel, four varieties did not pass our arbitrary minimum threshold of 0.05 (Fig. 4b). Spring barley Class, and winter barley KWS Joy, Mackie and Angora all had distances of 0.04 with their previously submitted parents Prestige, Wintmalt and KWS Tower and full sib Melanie, respectively. Since only 4 out of a total of 326,836 pairwise comparisons had a distance below the minimum threshold, it is not possible to visualise them in Fig. 4b. In addition, of these 4 pairs, Angora and Melanie were previously deemed indistinguishable in their DUS traits and had to be separated by either microsatellite markers ${ }^{23}$ or electrophoresis of hordein storage proteins ${ }^{24}$.

Ultimately, time and cost determine the feasibility of the current and alternative DUS methods. Here, we evaluated four methods: (1) morphological trait DUS ${ }^{25}$, (2) speed DUS ${ }^{26},(3)$ trait-specific marker DUS ${ }^{13}$, and (4) genomic DUS. Among these methods, the current DUS system with morphological trait takes the longest time as it usually requires one to two years of field or glasshouse trials. Recently, Jamali et al. ${ }^{26}$ proposed a speed DUS system by combining the current system with speed breeding ${ }^{27}$. However, this proposal requires experimental validations for every DUS trait since speed breeding alters plant development and many of the DUS traits are indeed developmental features. Both trait-specific marker and genomic marker methods require least amount of time, and it is possible to shorten the time to days provided there

212 is a routine demand. From the monetary aspect, both trait-specific and genomic marker methods 213 cost only a small fraction of the current DUS trait method. Trait-specific markers using Kompetitive 214 Allele Specific PCR (KASP) cost approximately $£ 11$ for 100 markers $^{28}$ while genomic markers 215 using the barley 50k iSelect SNP array ${ }^{29}$ cost approximately $£ 40$ for over 40,000 markers ${ }^{30}$. While 216 no cost information is available for speed DUS, it is unlikely to be less than the current DUS trait 217 evaluation which costs $£ 1040$ per candidate variety ${ }^{31}$. Given all considerations, genomic markers 218 remain the best method forward for DUS.

Being in the genomic era, we have access to great genomic resources like the barley $50 \mathrm{k}$ 220 iSelect SNP array $^{29}$ for application in DUS. As an example, we have illustrated how genomic 221 markers can be used to evaluate distinctness, uniformity and stability of new varieties (Fig. 5). 222 Instead of relying on morphological trait differences from common knowledge varieties in the 223 reference panel, we can determine a distance threshold based on genomic markers that would 
224 allow us to decide if a variety is sufficiently distinct. By sampling multiple seeds (or multiple pools of seeds), we can also test for uniformity based on the distances among these seeds or pools. For instance, uniformity could be defined such that the distances among the seeds from a candidate variety cannot be more than its distances with common knowledge varieties. We can quantify stability by measuring the genomic heterogeneity of the variety seed pool since a fully homogenous seed pool ensures genomic stability in subsequent generations of seed production. In an inbred species, this can be achieved by checking for genomic heterogeneity between seeds in the initial DUS application and final commercial seed lot. In an outcrossing species, this could be done by evaluating the change in allele frequencies between the initial and final seed lots after accounting for possible genomic drift. Overall, genomic markers provide a robust and effective option for improving DUS testing.

\section{Discussion}

Our analysis on the current DUS system using UK barley as an example has shown that morphological traits are not fit for DUS purposes. The trait combinatorial space gets narrower over time, and is likely worse in crop species with limited genetic variation. DUS traits with low heritabilities are not replicable outside the DUS trial and hence these traits have limited meaning to variety fingerprinting. As a consequence, there is no easy way for farmers to verify the identities of the varieties sown in their field. Genetic correlations between DUS and yield are detrimental to crop breeding due to the constraints imposed on selecting for higher yield and away from the common DUS trait combinatorial space. Besides, the current DUS process is time consuming and costly, which is non-ideal for small breeding companies. Unfortunately, alternatives like traitspecific markers and small marker sets are inadequate for DUS.

It is evident that the current DUS system is due for an update and we have shown that genomic markers are the best way forward. Aside from being able to address various shortcomings in the current system, it also opens up opportunities for bringing molecular editing into breeding practices and clarifies the boundary between new and essentially derived varieties.

251 Genome-edited varieties can be traced back, while remain superior in agronomic performances,

252 to their original non-edited varieties. Given the role of the DUS system in granting varietal rights, 253 it is the perfect setup for addressing the lack of genetic diversity in modern crop which threatens 254 food security ${ }^{46}$. This, obviously, is only possible with genomic markers. In addition, with the 255 impacts from Brexit (in the UK and EU) and Covid-19 looming for an unforeseeable future, there 
256 may be heavy restrictions on seed movement that impede the process of getting varieties into the

257 market. Such limitations are non-ideal since only a small fraction of the candidate varieties ends

258 up passing the DUS test while the rest ends up as a waste of time and money. With genomic

259 markers for DUS, it is trivial for testing centres to either receive DNA samples from breeders or

260 marker data from another testing centre in a different country. Lastly, genomic DUS will unlock a

261 new opportunity for an improved seed certification system to better protect breeders, farmers and

262 customers.

263

264 Acknowledgement

265 We thank the IMPROMALT consortium, especially Paul Shaw, Hazel Bull and Malcolm Macaulay,

266 for sharing the marker data for all barley varieties analysed here, as well as Rajiv Sharma, Ian

267 Dawson and David Marshall for helpful discussion throughout the work. 


\section{Methods}

269 DUS trait and marker data. DUS trait data from the UK national list were downloaded from the 270 National Institute of Agricultural Botany (NIAB) and Science and Advice for the Scottish 271 Agriculture (SASA) websites on $30^{\text {th }}$ April 2020. NIAB data is available at 272 https://www.niab.com/uploads/files/Spring_Barley_Descriptions_2019_V1.pdf and 273 https://www.niab.com/uploads/files/Winter_Barley_Descriptions_2019_V1.pdf while SASA data 274 is available at https://barley.agricrops.org/varieties. The NIAB data had a total of 287 barley varieties and the SASA data had a total of 728 varieties. Additional DUS trait data from NIAB were taken from Cockram et al. ${ }^{7}$, which had 577 varieties. After merging the different sources of NIAB data, there were 827 varieties remain. The original DUS trait data were stored as text descriptions and had to be converted into numerical scales using the key provided by APHA ${ }^{25}$. Both NIAB and SASA data had all 28 DUS traits, although some of the traits had high missing rate especially in the SASA data. Therefore, we used the NIAB data for our primary analyses and the SASA data for only comparative analysis between the two. While we attempted to source as many varieties with DUS trait data as possible, we did not have an exhaustive list of all UK barley varieties to date as we were limited to those which are available publicly.

Marker data from the UK national list were obtained from the IMPROMALT project (http://www.barleyhub.org/projects/impromalt/). The original marker data contained 809 varieties with 43,799 SNP markers genotyped on the barley 50k iSelect SNP array ${ }^{29}$. This contrasts with a previous DUS study by Cockram et al. ${ }^{7}$ which had 500 varieties and 1,536 SNP markers. Since a large proportion of the markers did not have any missing data, we removed any marker with missing data which left us with 40,078 SNP markers. In addition, we also obtained year of national listing and pedigree information of all varieties from the IMPROMALT project. Within these 809 varieties, 432 are spring barley, 372 are winter barley and 5 are alternative barley. We removed four varieties that did not have any Application for Protection (AFP) number, which left us with 805 varieties. Since there are only few alternative barley varieties, we excluded them from any analysis that requires separation of the data by seasonal types. The trait and marker data were merged by their AFP numbers. Unlike the variety names that are occasionally recycled, the AFP numbers are unique for each variety. They are also ordered by date of submission for DUS testing. Overall, we had 710 varieties that are in common between the DUS trait and marker data, which serves as our primary data for analysis.

299 DUS trait comparative analysis. We calculated the DUS trait discrepancies between NIAB and 
on a scale with an increment of 1, except for trait 3, 23 and 26 which were scored as either 1 or 9. To maintain a fair comparison across all traits, we converted those trait scores from 1 or 9 to 1 or 2. All DUS trait comparisons were performed only when there is complete pairwise data between NIAB and SASA.

Additionally, we subset the DUS trait data into spring and winter barley respectively to calculate the change in trait combinatorial space over time. This analysis was done by first sorting the barley varieties by their AFP number. Next, we computed the rolling mean of 20 varieties' Manhattan distances using dist function in $\mathrm{R}^{47}$ with an increment of one variety at a time. The lower the mean distance, the narrower the trait combinatorial space.

Univariate mixed linear model analyses of DUS traits. By leveraging the genomic relationship among the varieties, we partitioned the DUS phenotypic variance into additive genetic and residual variances using mmer function in the "sommer" package ${ }^{48}$ in $\mathrm{R}^{47}$. Briefly, the mixed model is described as $y=X \beta+g+e$. For any DUS trait with $n$ varieties, $y$ is an $n \times 1$ vector of DUS trait, $X$ is an $n \times n$ incidence matrix relating to fixed effects $\beta, \beta$ is an $n \times m$ matrix of $m$ fixed effects, $g$ is an $n \times 1$ vector of random additive genetic effect and $e$ is an $n \times 1$ vector of residual effect. The $m$ fixed effects included intercept, year of entry into national listing, and seasonal type, although the last effect was dropped when spring and winter barley datasets were analysed separately. The random additive genetic effect $g$ was restricted to a normal distribution of mean 0 and variance $\sigma_{g}{ }^{2} A$, where $\sigma_{g}{ }^{2}$ is the additive genetic variance and $A$ is an $n \times n$ additive genetic relationship matrix calculated using A.mat function in "sommer". Similarly, the residual effect followed a normal distribution of mean 0 and variance $\sigma_{e}{ }^{2} I$, where $\sigma_{e}{ }^{2}$ is the residual variance and I is an $n \times n$ identity matrix. For every DUS trait, we fitted the model using data from the spring barley dataset $(n=370)$, winter barley dataset $(n=335)$ and combined dataset $(n=710)$. We then extracted the genetic $\left(\sigma_{g}{ }^{2}\right)$ and phenotypic $\left(\sigma_{g}^{2}+\sigma_{e}{ }^{2}\right)$ variances and calculated heritabilities as $\sigma_{g}{ }^{2} /\left(\sigma_{g}{ }^{2}+\sigma_{e}{ }^{2}\right)$. yield data for spring barley from Mackay et al. ${ }^{19}$ and the Agriculture and Horticulture Development Board (AHDB) website for 509 varieties that were included in the VCU trials from 1948 to 2019. These varieties were trialled in multiple environments and years. The dry matter yield data from 1983 and onwards were taken from fungicide treated trials, and the data prior to that were taken from "best local practice" trials which meant that fungicide usage was left to the discretion of managers at each trial. To account for this difference, we created a "management" variable.

332 Varieties from 1983 and onwards were scored as 1 and the varieties prior to that were scored as 3330 for this variable. 
The raw dry matter yield data were fitted into a mixed linear model using Imer function in the "Ime4" package ${ }^{49}$ in $\mathrm{R}^{47}$. Briefly, the raw dry matter yield was set as the response variable, with variety as fixed effects, and management, management-by-year, management-by-year-byvariety and management-by-year-by-location as random effects. Next, we calculated the best linear unbiased estimates (BLUEs) for yield using the emmeans function in "emmeans" package ${ }^{50}$ 339 in $\mathrm{R}^{47}$.

Bivariate mixed linear model analyses of DUS traits and yield. We merged the DUS traits and yield data by the variety AFP numbers, which left us with 192 spring barley varieties in common. Unfortunately, we did not have access to older winter barley dry matter yield data, so the analysis here is only limited to spring barley. Similar to the univariate analyses, we fitted each DUS trait and dry matter yield BLUE into a mixed linear model using mmer function in "sommer" package ${ }^{48}$ in $\mathrm{R}^{47}$. Briefly, the bivariate models can be written as $y_{1}=X \beta+g_{1}+e_{1}$ and $y_{2}=X \beta+g_{2}+e_{2}$. For any pair of DUS trait and yield with $n$ varieties, $y_{1}$ is an $n \times 1$ vector of DUS trait, $y_{2}$ is an $n \times 1$ vector of yield, $X$ is an $n \times n$ incidence matrix relating to fixed effects $\beta, \beta$ is an $n \times m$ matrix of $m$ fixed effects, $g_{1}$ is an $n \times 1$ vector of random additive genetic effect for DUS trait, $g_{2}$ is an $n \times 1$ vector of random additive genetic effect for yield, $e_{1}$ is an $n \times 1$ vector of residual effect for DUS trait and $e_{2}$ is an $n \times 1$ vector of residual effect for yield. The $m$ fixed effects included intercept and year of entry into national listing. Unlike the univariate analyses, here the random additive genetic effect $g_{1}$ and $g_{2}$ were restricted to a multivariate normal distribution of mean 0 and variance $\left|\begin{array}{cc}\sigma_{g 1}^{2} & \rho_{g} \sigma_{g 1} \sigma_{g 2} \\ \rho_{g} \sigma_{g 1} \sigma_{g 2} & \sigma_{g 2}^{2}\end{array}\right| \otimes \mathrm{A}$, where $\sigma_{g 1}{ }^{2}$ is the additive genetic variance for DUS trait, $\sigma_{g 2}{ }^{2}$ is the additive genetic variance for yield, $\rho_{g}$ is the additive genetic correlation between DUS trait and yield, $\otimes$ is a Kronecker product and $A$ is an $n \times n$ additive genetic relationship matrix calculated using A.mat function in "sommer". Similarly, the residual effect followed a multivariate normal distribution of mean 0 and variance $\left|\begin{array}{cc}\sigma_{e 1}^{2} & \rho_{e} \sigma_{e 1} \sigma_{e 2} \\ \rho_{e} \sigma_{e 1} \sigma_{e 2} & \sigma_{e 2}^{2}\end{array}\right| \otimes \mathrm{I}$, where $\sigma_{e 1}{ }^{2}$ is the residual variance for DUS trait, $\sigma_{\mathrm{e} 2}{ }^{2}$ is the residual variance for yield, $\rho_{\mathrm{e}}$ is the residual correlation between DUS trait and yield and $I$ is an $n \times n$ identity matrix. From the bivariate mixed models, we extracted the genetic correlation as $\rho_{g}$ and phenotypic correlation as $\left(\rho_{g} \sigma_{g 1} \sigma_{g 2}+\rho_{e} \sigma_{e 1} \sigma_{e 2}\right) /\left(\left(\sigma_{g 1}{ }^{2}+\sigma_{e 1}{ }^{2}\right)\left(\sigma_{g 2}{ }^{2}+\right.\right.$ $\left.\left.\sigma_{\mathrm{e} 2}^{2}\right)\right)^{1 / 2}$.

362 GWAS on DUS traits. We performed GWAS on each DUS trait using data from the spring barley dataset $(n=370)$, winter barley dataset $(n=335)$ and combined dataset $(n=710)$. We used a similar 
"sommer" package ${ }^{48}$ in $\mathrm{R}^{47}$. Briefly, the GWAS model is $y=X \beta+m_{i} k_{i}+g+e$, where $m_{i}$ is an $n \times 1$ vector of marker genotype, $k_{i}$ is the marker effect and $i$ is the marker index from one to the total number of markers. The other terms are the same as previously described in the univariate mixed linear model. We evaluated the GWAS results for significant markers by using a threshold of false discovery rate (FDR) of 0.05 , as determined from qvalue function in "qvalue" package ${ }^{51}$ in $\mathrm{R}^{47}$. Since barley is an inbreeding species, linkage disequilibrium (LD) can complicate GWAS results especially when there is a highly significant marker. Therefore, for any trait where the marker significance exceeded $-\log _{10}$ p of 10 , we performed a follow-up GWAS with the most significant marker as a fixed effect. The re-evaluation threshold was chosen as 10 to minimise the number of GWAS runs as we were only interested in identifying any potential peaks that are masked due to major segregating loci. If any of the markers on other chromosomes were initially significant due to LD with the causative locus, then these markers should drop below the significance threshold in the second GWAS.

Evaluation on the usefulness of small marker set in DUS via simulation. To evaluate the 45 DUS markers in Owen et al. ${ }^{21}$, we simulated these markers in the progeny of known parent pairs. We used 39 out of the 45 markers for simulation as six of the markers were either absent or low quality in our dataset. Based on the pedigree information, there were 212 varieties with marker data available for their parents and these varieties were generated from intercross between the parents. For each variety and its parents, we simulated 10,000 $F_{6}$ progeny using "AlphaSimR" package ${ }^{52}$ in $\mathrm{R}^{47}$. We then compared the simulated progeny to the known progeny (variety) and its two parents, and counted the number of exact matches in the DUS markers. Additionally, we bootstrapped the comparisons for 1,000 times to get a better estimate of the mean count of exact matches. For comparison within the simulated progeny, we tabulated the number of occurrences of each progeny with unique DUS marker haplotype.

Comparing Manhattan distances from DUS traits against different number of markers. To evaluate the number of markers needed for DUS, we randomly sampled one to the maximum number of markers with an increment of $\log _{10}$ of 0.1 . We then calculated the Manhattan distances from DUS traits and markers using dist function in $\mathrm{R}^{47}$. For each set of markers, we computed the correlation between the Manhattan distances from DUS traits and marker data. In addition, we also separated the DUS traits into a high heritability group $\left(h^{2}>0.5\right)$ and low heritability group $\left(h^{2}\right.$ $<0.5)$, and computed the correlations similarly.

396 Demonstrating the use of genomic markers in DUS via simulation. To test how genomic 397 markers can be used in DUS, we chose two known spring barley parent pairs with low and high 
398 genomic distances. Acumen's parents, Propino and Quench with a distance of 0.20 represents 399 the low distance option, while Berwick's parents, Riviera and Cooper with a distance of 0.59 400 represents the high distance option. From each of these parent pairs, we simulated 1,000 $F_{6}$ and $401 \mathrm{BC}_{1} \mathrm{~S}_{4}$ progeny using the "AlphaSimR" package ${ }^{52}$ in $\mathrm{R}^{47}$. We then computed the Manhattan 402 distances from each simulated progeny group using dist function in $\mathrm{R}^{47}$.

403 Data availability. The compiled DUS trait data from NIAB and SASA and BLUEs for dry matter 404 yield are available in Supplementary File 1. The IMPROMALT marker data is available at 405 http://www.barleyhub.org/projects/impromalt/. All source file website links have been archived at 406 https://web.archive.org/. The R scripts for all analyses are available at https://github.com/cjyang407 sruc/DUS. 
408 Table 1. DUS trait names and heritabilities, standard errors in parentheses.

\begin{tabular}{|c|c|c|c|c|}
\hline \multirow{2}{*}{ Trait } & \multirow{2}{*}{ Name } & \multicolumn{3}{|c|}{$h^{2}$} \\
\hline & & Combined & Spring & Winter \\
\hline 1 & kernel: colour of aleurone layer & $0.78(0.04)$ & $0.16(0.06)$ & $0.79(0.06)$ \\
\hline 2 & plant: growth habit & $0.25(0.05)$ & $0.17(0.06)$ & $0.24(0.07)$ \\
\hline 3 & lowest leaves: hairiness of leaf sheaths & $0.75(0.04)$ & NA & $0.69(0.07)$ \\
\hline 4 & flag leaf: intensity of anthocyanin colouration of auricles & $0.74(0.05)$ & $0.19(0.06)$ & $0.84(0.08)$ \\
\hline 5 & flag leaf: attitude & $0.28(0.13)$ & $0.28(0.19)$ & $0.25(0.16)$ \\
\hline 6 & flag leaf: glaucosity of sheath & $0.12(0.04)$ & $0.05(0.03)$ & $0.10(0.05)$ \\
\hline 7 & time of ear emergence (first spikelet visible on $50 \%$ of ears) & $0.28(0.05)$ & $0.20(0.06)$ & $0.26(0.07)$ \\
\hline 8 & awns: intensity of anthocyanin colouration of tips & $0.67(0.05)$ & $0.09(0.04)$ & $0.83(0.08)$ \\
\hline 9 & ear: glaucosity & $0.42(0.05)$ & $0.45(0.07)$ & $0.33(0.08)$ \\
\hline 10 & ear: attitude & $0.25(0.05)$ & $0.26(0.07)$ & $0.17(0.06)$ \\
\hline 11 & plant: length (stem, ear and awns) & $0.17(0.04)$ & $0.13(0.05)$ & $0.14(0.06)$ \\
\hline 12 & ear: number of rows & $1.00(0.01)$ & NA & $1.00(0.03)$ \\
\hline 13 & ear: shape & $0.10(0.04)$ & $0.04(0.03)$ & $0.09(0.05)$ \\
\hline 14 & ear: density & $0.23(0.05)$ & $0.14(0.05)$ & $0.24(0.07)$ \\
\hline 15 & ear: length (excluding awns) & $0.18(0.05)$ & $0.05(0.04)$ & $0.29(0.08)$ \\
\hline 16 & awn: length (compared to ear) & $0.18(0.04)$ & $0.15(0.05)$ & $0.11(0.05)$ \\
\hline 17 & rachis: length of first segment & $0.34(0.05)$ & $0.32(0.07)$ & $0.28(0.07)$ \\
\hline 18 & rachis: curvature of first segment & $0.26(0.05)$ & $0.25(0.07)$ & $0.20(0.07)$ \\
\hline 19 & ear: development of sterile spikelets & $1.00(0.04)$ & $1.00(0.06)$ & $1.00(0.09)$ \\
\hline 20 & sterile spikelets: attitude (in mid-third of ear) & $0.64(0.06)$ & $0.63(0.08)$ & $0.49(0.10)$ \\
\hline 21 & median spikelet: length of glume and its awn relative to grain & $0.15(0.04)$ & $0.07(0.04)$ & $0.18(0.06)$ \\
\hline 22 & grain: rachilla hair type & $1.00(0.01)$ & $1.00(0.02)$ & $0.84(0.05)$ \\
\hline 23 & grain: husk & $0.01(0.02)$ & $0.04(0.03)$ & $0.00(0.02)$ \\
\hline 24 & grain: anthocyanin colouration of nerves of lemma & $0.69(0.05)$ & $0.31(0.07)$ & $0.78(0.07)$ \\
\hline 25 & $\begin{array}{l}\text { grain: speculation of inner lateral nerves of dorsal side of } \\
\text { lemma }\end{array}$ & $0.74(0.04)$ & $0.49(0.07)$ & $0.78(0.06)$ \\
\hline 26 & grain: hairiness of ventral furrow & $0.96(0.02)$ & $0.65(0.07)$ & $0.94(0.04)$ \\
\hline 27 & grain: disposition of lodicules & $0.91(0.02)$ & $0.99(0.03)$ & NA \\
\hline 28 & seasonal type & $1.00(0.00)$ & NA & NA \\
\hline
\end{tabular}


bioRxiv preprint doi: https://doi.org/10.1101/2020.10.08.331892; this version posted October 9, 2020. The copyright holder for this preprint

410 Table 2. GWAS results. Significant GWAS peaks (FDR < 0.05) are summarised here along 411 with their closest known gene or locus.

\begin{tabular}{|c|c|c|c|c|c|c|c|c|c|c|}
\hline \multirow{2}{*}{ Trait } & \multicolumn{5}{|c|}{ GWAS peak ${ }^{a}$} & \multicolumn{3}{|c|}{ Allele frequency } & \multicolumn{2}{|l|}{ Known gene/locus } \\
\hline & Chr & Pos (Mb) & Effect & $-\log _{10} p$ & Pop ${ }^{b}$ & $\mathrm{C}$ & $\mathrm{S}$ & W & Name & Pos (Mb) \\
\hline 1 & $4 \mathrm{H}$ & 525.07 & 0.74 & 96.02 & $\mathrm{C}, \mathrm{S}, \mathrm{W}$ & 0.19 & 0.02 & 0.37 & $\mathrm{MbHF} 5^{\mathrm{c}}(\mathrm{B} \mid \mathrm{x} 1)^{32}$ & 534.04 \\
\hline 2 & $3 \mathrm{H}$ & 631.83 & -0.69 & 6.40 & C & 0.49 & 0.05 & 0.98 & HORVU3Hr1G090910d,33 & 633.53 \\
\hline 3 & $1 \mathrm{H}$ & 473.27 & -0.25 & 4.92 & $\mathrm{C}$ & 0.16 & 0.01 & 0.32 & NA & NA \\
\hline 3 & $4 \mathrm{H}$ & 631.68 & 3.32 & 116.93 & $\mathrm{C}, \mathrm{W}$ & 0.44 & 0.00 & 0.91 & $\begin{array}{l}\text { HORVU4Hr1G085920d, } \\
\text { HORVU4Hr1G085590 } \\
\text { (Hsh1 })^{20}\end{array}$ & 633.03 \\
\hline 4 & $2 \mathrm{H}$ & 676.76 & -2.25 & 42.77 & $\mathrm{C}, \mathrm{W}$ & 0.25 & 0.00 & 0.53 & HORVU2Hr1G096810 (Ant2) & 676.85 \\
\hline 4 & $7 \mathrm{H}$ & 73.55 & -0.80 & 10.67 & $\mathrm{C}$ & 0.10 & 0.02 & 0.19 & HORVU7Hr1G034630 (Ant1) & 72.92 \\
\hline 8 & $2 \mathrm{H}$ & 675.76 & -2.27 & 55.97 & $\mathrm{C}, \mathrm{W}$ & 0.25 & 0.00 & 0.53 & HORVU2Hr1G096810 (Ant2) ${ }^{7}$ & 676.85 \\
\hline 8 & $6 \mathrm{H}$ & 536.07 & 0.57 & 6.75 & $S$ & 0.30 & 0.16 & 0.45 & NA & NA \\
\hline 8 & $7 \mathrm{H}$ & 73.55 & -0.69 & 11.01 & $\mathrm{C}, \mathrm{W}$ & 0.10 & 0.02 & 0.19 & HORVU7Hr1G034630 (Ant1) & 72.92 \\
\hline 9 & $1 \mathrm{H}$ & 0.29 & -0.52 & 6.23 & $\mathrm{C}, \mathrm{S}, \mathrm{W}$ & 0.07 & 0.07 & 0.06 & EAR-G_135 & $0.50^{\mathrm{e}}$ \\
\hline 9 & $2 \mathrm{H}$ & 6.18 & -0.33 & 6.98 & C & 0.43 & 0.52 & 0.34 & NA & NA \\
\hline 11 & $4 \mathrm{H}$ & 608.43 & -0.30 & 5.93 & $\mathrm{C}$ & 0.45 & 0.49 & 0.41 & NA & NA \\
\hline 12 & $2 \mathrm{H}$ & 663.88 & 0.04 & 6.05 & $\mathrm{C}, \mathrm{W}$ & 0.30 & 0.24 & 0.37 & HORVU2Hr1G092290 (Vrs1) & 651.03 \\
\hline 12 & $5 \mathrm{H}$ & 579.73 & -0.04 & 6.11 & $\mathrm{C}$ & 0.42 & 0.00 & 0.89 & HORVU5Hr1G081450 (Vrs2) $)^{37}$ & 564.41 \\
\hline 13 & $3 \mathrm{H}$ & 437.24 & 0.63 & 5.56 & C & 0.05 & 0.00 & 0.11 & NA & NA \\
\hline 15 & $4 \mathrm{H}$ & 608.38 & -0.22 & 4.76 & S & 0.45 & 0.49 & 0.41 & $45^{38}$ & $618.00^{\mathrm{e}}$ \\
\hline 19 & $2 \mathrm{H}$ & 652.42 & -0.49 & 146.59 & $\mathrm{C}, \mathrm{S}, \mathrm{W}$ & 0.29 & 0.24 & 0.34 & HORVU2Hr1G092290 (Vrs1) & 651.03 \\
\hline 20 & $1 \mathrm{H}$ & 404.92 & -0.38 & 11.39 & $\mathrm{C}, \mathrm{S}, \mathrm{W}$ & 0.30 & 0.02 & 0.60 & HORVU1Hr1G051010 (Vrs3) & 378.41 \\
\hline 20 & $2 \mathrm{H}$ & 655.81 & -0.63 & 19.19 & $\mathrm{C}, \mathrm{S}, \mathrm{W}$ & 0.22 & 0.20 & 0.25 & HORVU2Hr1G092290 (Vrs1) & 651.03 \\
\hline 20 & $3 \mathrm{H}$ & 659.54 & -0.19 & 4.12 & $\mathrm{C}$ & 0.16 & 0.01 & 0.32 & NA & NA \\
\hline 20 & $5 \mathrm{H}$ & 488.46 & -0.16 & 4.37 & $\mathrm{C}$ & 0.06 & 0.02 & 0.11 & NA & NA \\
\hline 21 & $7 \mathrm{H}$ & 47.56 & 0.17 & 7.93 & $\mathrm{C}, \mathrm{W}$ & 0.08 & 0.07 & 0.08 & NA & NA \\
\hline 22 & $5 \mathrm{H}$ & 542.50 & -0.16 & 24.72 & $\mathrm{C}, \mathrm{S}, \mathrm{W}$ & 0.36 & 0.52 & 0.18 & $\mathrm{Srh}^{7}$ & $547.24^{\mathrm{e}}$ \\
\hline 23 & $7 \mathrm{H}$ & 612.52 & -0.31 & 6.71 & $\mathrm{C}, \mathrm{S}$ & 0.06 & 0.10 & 0.02 & HORVU7Hr1G089930 (Nud) ${ }^{41}$ & 546.59 \\
\hline 24 & $2 \mathrm{H}$ & 676.20 & -1.45 & 44.08 & $\mathrm{C}, \mathrm{S}, \mathrm{W}$ & 0.34 & 0.14 & 0.56 & HORVU2Hr1G096810 (Ant2) ${ }^{7}$ & 676.85 \\
\hline 24 & $7 \mathrm{H}$ & 72.97 & -0.56 & 8.46 & $\mathrm{C}, \mathrm{W}$ & 0.22 & 0.03 & 0.43 & HORVU7Hr1G034630 (Ant1) & 72.92 \\
\hline 25 & $2 \mathrm{H}$ & 638.37 & 1.97 & 57.54 & $\mathrm{C}, \mathrm{S}, \mathrm{W}$ & 0.17 & 0.09 & 0.26 & Gth1 ${ }^{7}$ & $647.46^{\mathrm{e}}$ \\
\hline 26 & $6 \mathrm{H}$ & 0.33 & 3.85 & 152.61 & $\mathrm{C}, \mathrm{W}$ & 0.14 & 0.00 & 0.29 & $11 \_20881^{7}$ & $5.20^{\mathrm{e}}$ \\
\hline 27 & $2 \mathrm{H}$ & 724.71 & -0.10 & 24.45 & $\mathrm{C}, \mathrm{S}$ & 0.48 & 0.05 & 0.95 & HORVU2Hr1G113880 (Cly1) ${ }^{42}$ & 730.03 \\
\hline 28 & $1 \mathrm{H}$ & 511.92 & -0.51 & 47.74 & $\mathrm{C}$ & 0.48 & 0.00 & 1.00 & HORVU1Hr1G076430 (Ppd-H2) ${ }^{43}$ & 514.1 \\
\hline 28 & $4 \mathrm{H}$ & 643.68 & -0.63 & 63.95 & $\mathrm{C}$ & 0.48 & 0.00 & 1.00 & Vrn- $-2^{44}$ & NA \\
\hline 28 & $5 \mathrm{H}$ & 571.03 & -0.34 & 38.29 & $\mathrm{C}$ & 0.46 & 0.00 & 0.98 & HORVU5Hr1G095630 (Vrn-H1) ${ }^{45}$ & 599.09 \\
\hline
\end{tabular}

${ }^{a}$ If the GWAS peak is found in more than one population, only the results from the combined $(C)$ analysis are shown here.

$414 \quad{ }^{b}$ This column indicates which populations (C: Combined, S: Spring, W: Winter) showed significance for any given GWAS peak.

$415{ }^{c}$ MbHF35 is a cluster of 3 linked genes: HvMYB4H (HORVU4Hr1G063760), HvMYC4H (NA) and HvF35H (HORVU4Hr1G063780).

416 dunverified candidate genes.

417 eApproximated physical positions based on genetic positions. 

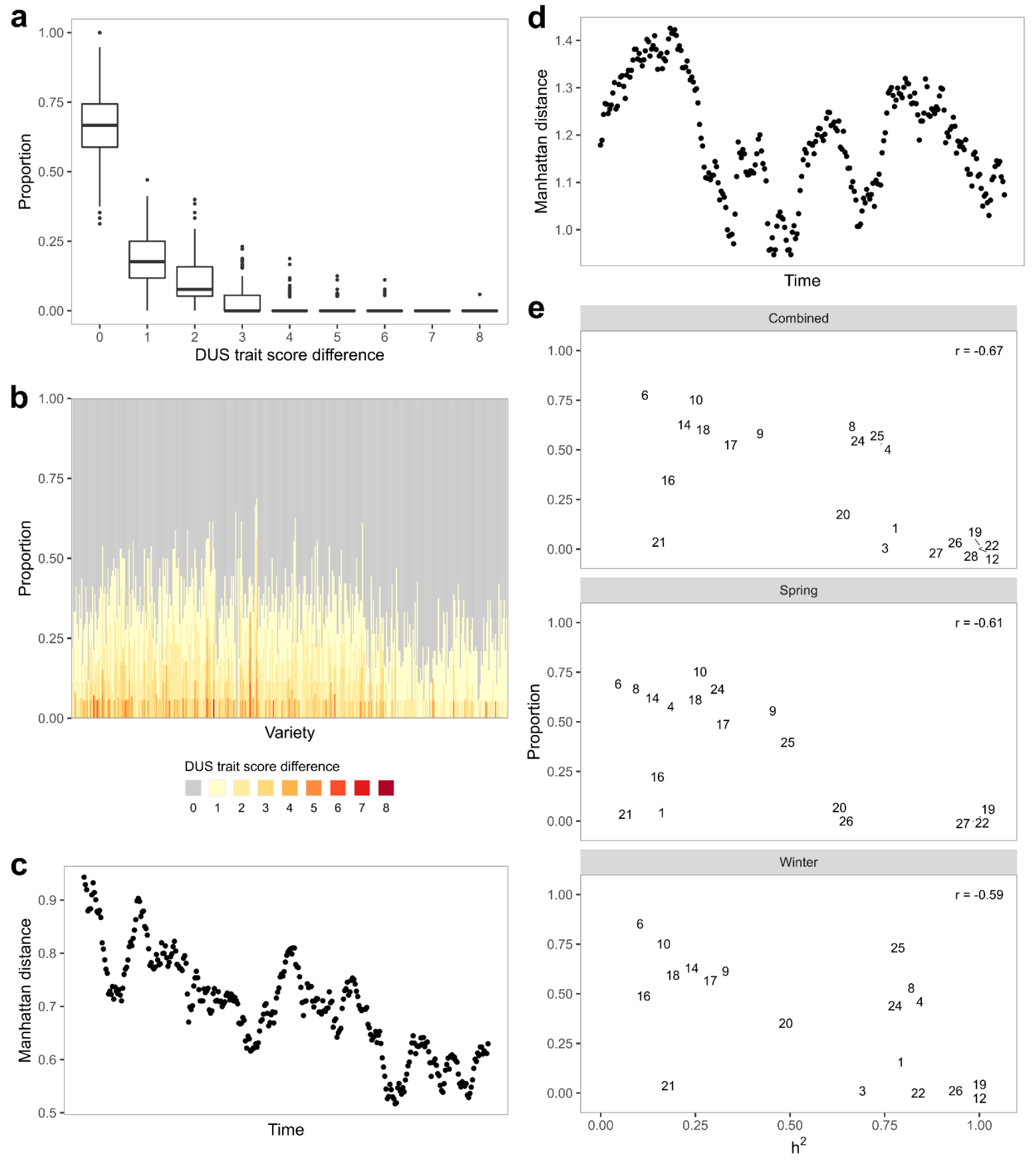

Fig. 1. DUS trait discrepancies and combinatorial space. a, Boxplots of proportion of DUS trait score differences between NIAB and SASA data. $\mathbf{b}$, Proportion of DUS trait score differences for each variety, with oldest variety (1963) on the left and newest variety (2007) on the right. c, Rolling mean distances of 20 spring barley varieties calculated from DUS traits with an increment of one new variety at a time. Leftmost point on the "Time" axis indicates the mean from 20 earliest varieties, while rightmost point indicates the mean from 20 latest varieties. d, Rolling mean distances of 20 winter barley varieties calculated from DUS traits with an increment of one new variety at a time. e, Relationships between proportion of DUS trait score differences and heritabilities, separated by all (spring and winter combined), spring only and winter only groups. Each point is shown as its trait number, which is available in Table 1. 
bioRxiv preprint doi: https://doi.org/10.1101/2020.10.08.331892; this version posted October 9, 2020. The copyright holder for this preprint (which was not certified by peer review) is the author/funder, who has granted bioRxiv a license to display the preprint in perpetuity. It is made available under aCC-BY-NC-ND 4.0 International license.

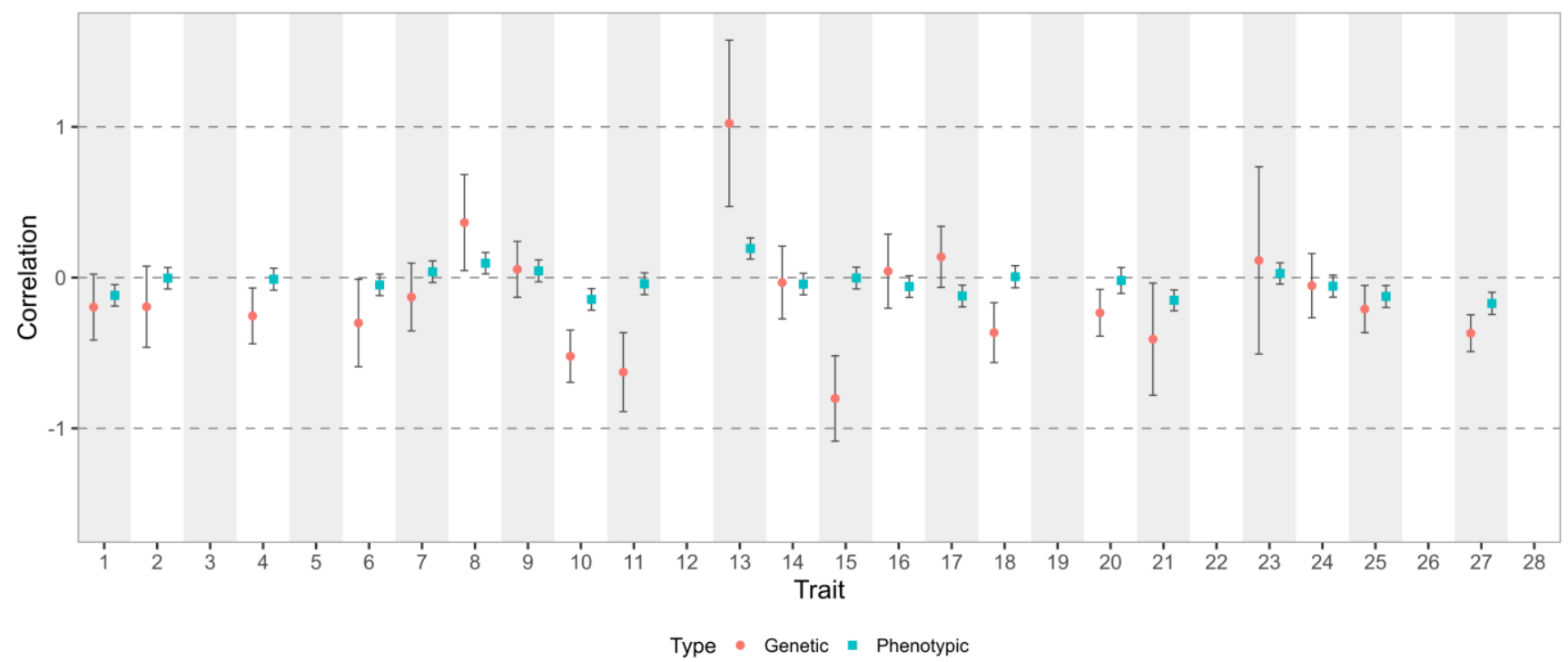

429 Fig. 2. Genetic and phenotypic correlations between DUS traits and yield. Genetic and phenotypic correlations, along with their error bars, are shown for each DUS trait and yield. No correlation is available for trait $3,5,12,19,22,26$ and 28 because of either high missing rate or lack of variation in the DUS trait. 

available under aCC-BY-NC-ND 4.0 International license.
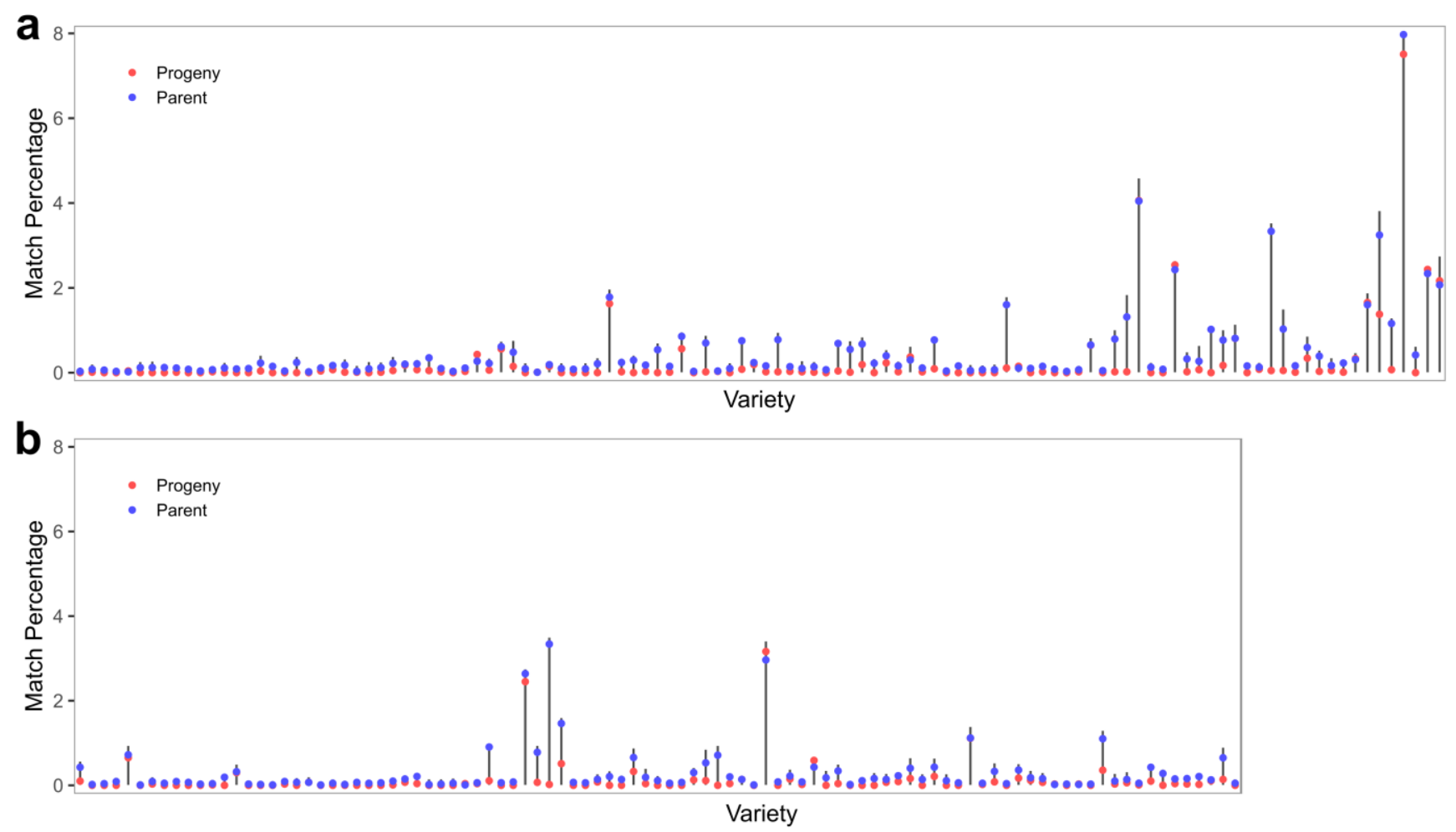

433 Fig. 3. Usefulness of a small marker set for DUS. This small marker set is taken from 39 DUS markers in and parents. Grey vertical bars represent the spread of match percentage among the simulated progeny. a, 114 spring barley varieties sorted from oldest (left, 1982) to newest (right, 2016). b, 97 winter barley varieties sorted from oldest (left, 1987) to newest (right, 2016). 
bioRxiv preprint doi: https://doi.org/10.1101/2020.10.08.331892; this version posted October 9,2020 . The copyright holder for this preprint (which was not certified by peer review) is the author/funder, who has granted bioRxiv a license to display the preprint in perpetuity. It is made available under aCC-BY-NC-ND 4.0 International license.
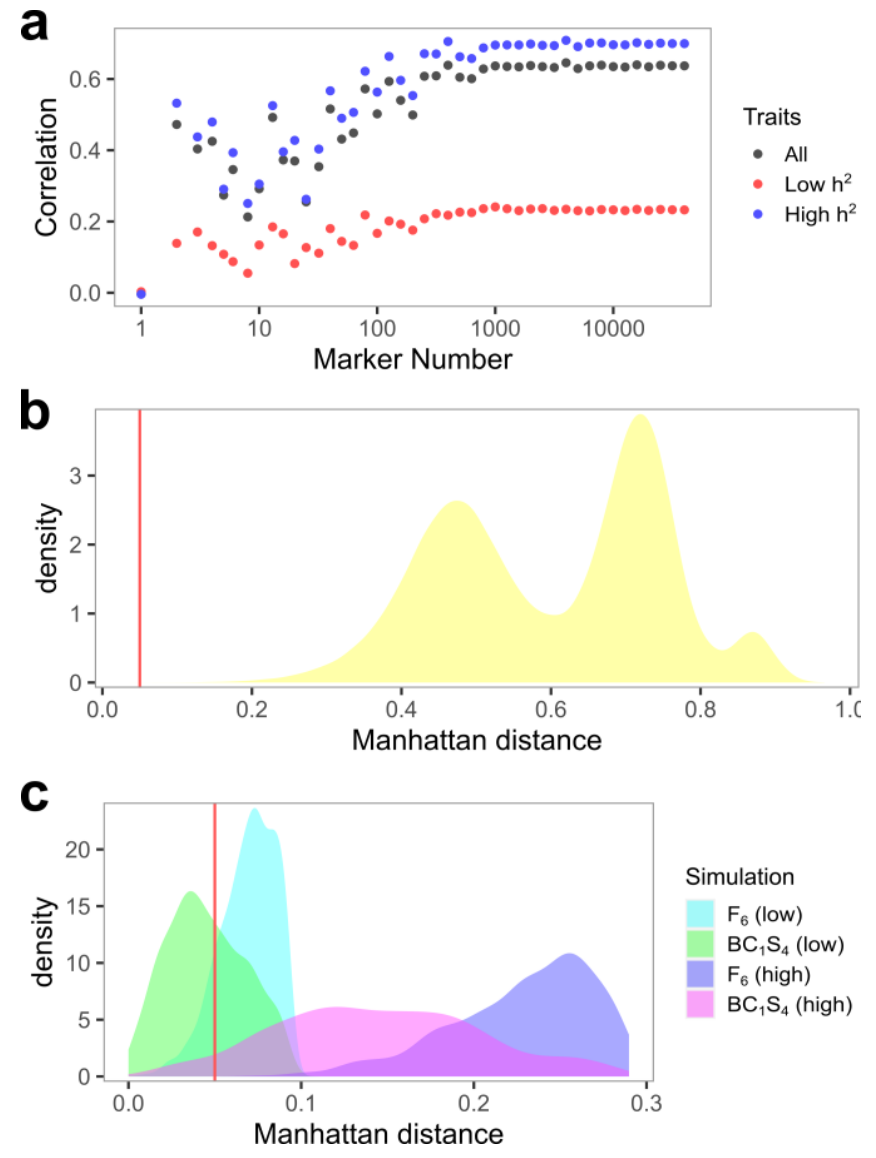

439 Fig. 4. Analyses of genomic markers for DUS. a, Correlation in Manhattan distances from DUS traits and various number of randomly sampled markers. b. Distribution of Manhattan distances among 805 barley varieties. Within seasonal type distances dominate the first left peak while between seasonal type distances dominate the two right peaks. Arbitrary distance threshold of 0.05 is marked with a red vertical line. c, Distribution of minimum Manhattan distances from each simulated progeny and reference panel (805 varieties). Similar arbitrary threshold of 0.05 is marked. 
DUS by morphological traits

DUS by genomic markers

\section{Distinctness}

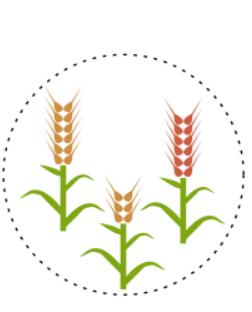

Reference panel

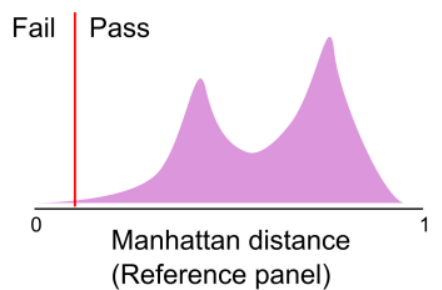

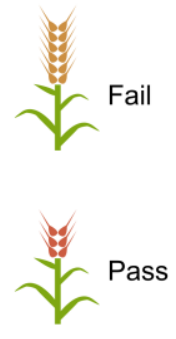

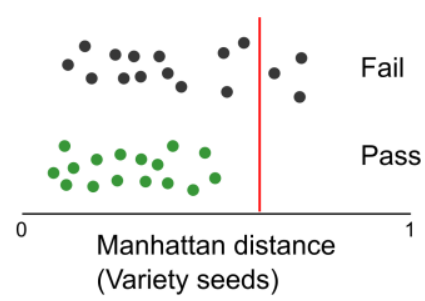

Uniformity

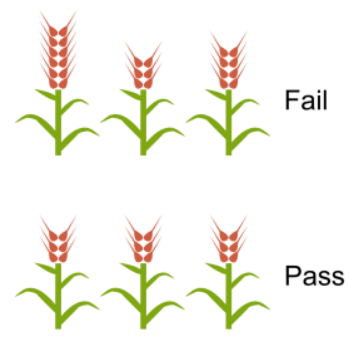

Manhattan distance Manhattan distance
(Reference panel)

Fig. 5. Simplified illustrations of the current DUS system and its potential successor. In the current system, distinctness, uniformity and stability of a candidate variety is determined by comparison of 28 morphological traits to a reference panel of common knowledge varieties and itself. We propose that an upgrade to the current system by using genomic markers instead of morphological traits. Here, distinctness is evaluated based on a minimum distance threshold, and anything below the threshold could be considered EDVs. Uniformity and stability evaluations are straightforward, as they each require the seeds to be close in distance to each other and low in genetic heterogeneity. 


\section{References}

454 1. Helfer, L. R. Intellectual property rights in plant varieties. International legal regimes and policy options for national governments. FAO Legislative Study 85, 1-113 (2004).

2. UPOV. General introduction to the examination of distinctness, uniformity and stability and the development of harmonized descriptions of new varieties of plants, TG/1/3. https://www.upov.int/en/publications/tg-rom/tg001/tg_1_3.pdf (2002).

3. Jamali, S. H., Cockram, J. \& Hickey, L. T. Insights into deployment of DNA markers in plant variety protection and registration. Theor. Appl. Genet. 132, 1911-1929 (2019).

4. Council of the European Union. Council directive 2002/53/EC of 13 June 2002 on the common catalogue of varieties of agricultural plant species. https://eur-lex.europa.eu/legalcontent/EN/ALL/?uri=celex:32002L0053 (2002).

5. Bailey-Serres, J., Parker, J. E., Ainsworth, E. A., Oldroyd, G. E. D. \& Shroeder, J. I. Genetic strategies for improving crop yield. Nature 575, 109-118 (2019).

6. Cooke, R. J. \& Reeves, J. C. Plant genetic resources and molecular markers: variety registration in a new era. Plant Genet. Res. 1, 81-87 (2003).

7. Cockram, J. et al. Genome-wide association mapping to candidate polymorphism resolution in the unsequenced barley genome. Proc. Natl. Acad. Sci. USA 107, 21611-21616 (2010).

8. Muthoni, J. \& Nyamongo, D. O. Seed systems in Kenya and their relationship to on-farm conservation of food crops. J. New Seeds 9, 330-342 (2008).

9. Jamnadass, R. et al. Enhancing African orphan crops with genomics. Nat. Genet. 52, 356-360.

10. Jones, et al. Evaluation of the use of high-density SNP genotyping to implement UPOV Model 2 for DUS testing in barley. Theor. Appl. Genet. 126, 901-911 (2013).

11. Nybom, H., Rogstad, S. H. \& Schaal, B. A. Genetic variation detected by use of the M13 "DNA fingerprint" probe in Malus, Prunus, and Rubus (Rosaceae). Theor. Appl. Genet. 79, 153-156.

12. Gunjaca, J. et al. Discriminating maize inbred lines using molecular and DUS data. Euphytica 161, 165-172 (2008).

13. Cockram, J., Jones, H., Norris, C. \& O'Sullivan, D. M. Evaluation of diagnostic molecular markers for DUS phenotypic assessment in the cereal crop, barley (Hordeum vulgare ssp. vulgare L.). Theor. Appl. Genet. 125, 1735-1749 (2012).

14. Pourabed, E. et al. Identification and DUS testing of rice varieties through microsatellite markers. Int. J. Plant Genom. 6, 965073 (2015).

15. Tian, H.-L. et al. Development of maizeSNP3072, a high-throughput compatible SNP array, for DNA fingerprinting identification of Chinese maize varieties. Mol. Breed. 35, 136 (2015).

16. Achard, F. \& Nelson, B. Evaluation of soybean molecular marker public resources for potential application in plant variety protection. 
488 17. UPOV. Guidance on the use of biochemical and molecular markers in the examination of distinctness, uniformity and stability (DUS). https://www.upov.int/edocs/tgpdocs/en/tgp_15.pdf (2019).

18. Donald, C. M. The breeding of crop ideotypes. Euphytica 17, 385-403 (1968).

19. Mackay, I. et al. Reanalyses of the historical series of UK variety trials to quantify the contributions of genetic and environmental factors to trends and variability in yield over time. Theor. Appl. Genet. 122, 225-238 (2011).

20. Saade, S. et al. A donor-specific QTL, exhibiting allelic variation for leaf sheath hairiness in a e0189446 (2017).

21. Owens, H., Pearson, K., Roberts, A. M. I., Reid, A. \& Russell, J. Single nucleotide polymorphism assay to distinguish barley (Hordeum vulgare L.) varieties in support of seed certification. Genet. Res. Crop Evol. 66, 1243-1256 (2019).

22. Lawson, C. Plant breeder's rights and essentially derived varieties: still searching for workable solutions. In 32 European intellectual property review 499, Griffith University Law School research paper no. 16-17. Griffith University, Queensland, Australia (2016).

23. Russell, J. et al. Discriminating between barley genotypes using microsatellite markers. Genome 40, 442-450 (1997).

24. Thomas, W. et al. Project report no. 528: Association genetics of UK elite barley (AGOUEB). HGCA (2014).

25. APHA. United Kingdom national list technical protocol for official examination of distinctness, uniformity and stability (DUS), barley, Hordeum vulgare L. sensu lato. https://assets.publishing.service.gov.uk/government/uploads/system/uploads/attachment_data/file/9 06760/dus-protocol-barley.pdf (2020).

26. Jamali, S. H., Cockram, J. \& Hickey, L. T. Is plant variety registration keeping pace with speed breeding techniques? Euphytica 216, 131 (2020).

27. Watson, A. et al. Speed breeding is a powerful tool to accelerate crop research and breeding. Nat. Plants 4, 23-29 (2018).

28. Ayalew, H. et al. Comparison of Taqman, KASP and rhAmp SNP genotyping platforms in hexaploid wheat. PLoS One 14, e0217222 (2019).

29. Bayer, M. M. et al. Development and evaluation of a barley 50k iSelect SNP array. Front. Plant Sci.
8, 1792 (2017).

30. Darrier, B. et al. A comparison of mainstream genotyping platforms for the evaluation and use of barley genetic resources. Front. Plant Sci. 10, 544 (2019). 
31. APHA. Fees for national listings (NL) and plant breeders rights (PBR).

https://assets.publishing.service.gov.uk/government/uploads/system/uploads/attachment_data/file/8

525 94729/pbr-fees.pdf (2018).

32. Jia, Y. et al. Uncovering the evolutionary origin of blue anthocyanins in cereal grains. Plant J. 101, 1057-1074 (2020).

33. Zhou, Y. et al. Towards the identification of a gene for prostrate tillers in barley (Hordeum vulgare
L.). PLoS One 13, e0192263 (2018).

34. Himi, E. \& Taketa, S. Isolation of candidate genes for the barley Ant1 and wheat Rc genes controlling anthocyanin pigmentation in different vegetative tissues. Mol. Genet. Genomics 290, 1287-1298 (2015).

35. Barral-Baron, G. Investigation and validation of QTL for yield and yield components in winter barley. PhD thesis, Newcastle University, Newcastle (2016).

36. Komatsuda, T. et al. Six-rowed barley originated from a mutation in a homeodomain-leucine zipper I-class homeobox gene. Proc. Natl. Acad. Sci. USA 104, 1424-1429 (2007).

37. Youssef, H. M. et al. VRS2 regulates hormone-mediated inflorescence patterning in barley. Nat. Genet. 49, 157-161 (2017).

38. Xu, X. et al. Genome-wide association analysis of grain yield-associated traits in a pan-European barley cultivar collection. Plant Genom. 11, 170073 (2018).

39. Sakuma, S. et al. Extreme suppression of lateral floret development by a single amino acid change in the VRS1 transcription factor. Plant Physiol. 175, 1720-1731 (2017).

40. Bull, H. et al. Barley SIX-ROWED SPIKE3 encodes a putative Jumonji C-type H3K9me2/me3 demethylase that represses lateral spikelet fertility. Nat. Comm. 8, 936 (2017).

41. Taketa, S. et al. Barley grain with adhering hulls is controlled by an ERF family transcription factor gene regulating a lipid biosynthesis pathway. Proc. Natl. Acad. Sci. USA 105, 4062-4067 (2008).

42. Nair, S. K. et al. Cleistogamous flowering in barley arises from the suppression of microRNA-guided HvAP2 mRNA cleavage. Proc. Natl. Acad. Sci. USA 107, 490-495 (2010).

43. Faure, S., Higgins, J., Turner, A. \& Laurie, D. A. The FLOWERING LOCUS T-like gene family in barley (Hordeum vulgare). Genetics 176, 599-609 (2007).

44. Dubcovsky, J., Chen, C. \& Yan, L. Molecular characterization of the allelic variation at the VRN-H2 vernalization locus in barley. Mol. Breed. 15, 395-407 (2005).

45. von Zitzewitz, J. et al. Molecular and structural characterization of barley vernalization genes. Plant Mol. Biol. 59, 449-467 (2005).

46. Esquinas-Alcázar, J. Protecting crop genetic diversity for food security: political, ethical and technical challenges, Nat. Rev. Genet. 6, 946-953 (2005).

47. R Core Team. R: a language and environment for statistical computing. R Foundation for Statistical Computing, Vienna, Austria. https://www.R-project.org (2020). 
bioRxiv preprint doi: https://doi.org/10.1101/2020.10.08.331892; this version posted October 9,2020 . The copyright holder for this preprint

(which was not certified by peer review) is the author/funder, who has granted bioRxiv a license to display the preprint in perpetuity. It is made available under aCC-BY-NC-ND 4.0 International license.

558 48. Covarrubias-Pazaran, G. Genome-assisted prediction of quantitative traits using the R package

559 sommer. PLoS One 11, e0156744 (2016).

560 49. Bates, D., Maechler, M., Bolker, B. \& Walker, S. Fitting linear mixed-effects models using Ime4. J

561 Stat. Softw. 67, 1-48 (2015).

562 50. Lenth, R. et al. emmeans: estimated marginal means, aka least-squares means. R package version 563 1.4.8. https://github.com/rvlenth/emmeans (2020).

564 51. Storey, J. D., Bass, A. J., Dabney, A., Robinson, D. \& Warnes, G. qvalue: Q-value estimation for

565 false discovery rate control. R package version 2.20.0. https://github.com/jdstorey/qvalue (2020).

566 52. Gaynor, R. C., Gorjanc, G. \& Hickey, J. M. AlphaSimR: an R-package for breeding program

567 simulations. Biorxiv. https://doi.org/10.11101/2020.08.10.245167 (2020). 